\title{
Modeling Brain Anatomy with 3D Arrangements of Curves
}

Washington Mio ${ }^{1}$, John C Bowers ${ }^{2}$, Monica K Hurdal' ${ }^{1}$, and Xiuwen Liü

${ }^{1}$ Department of Mathematics, Florida State University, Tallahassee, FL 32306-4510

${ }^{2}$ Department of Computer Science, Florida State University, Tallahassee, FL 32306-4530

\section{Introduction}

We employ $3 \mathrm{D}$ arrangements of curves to represent and analyze $3 \mathrm{D}$ shapes. The arrangements of curves may vary from fairly sparse - such as a collection of sulcal lines that coarsely approximates the shape of the brain - to very dense decompositions of the cortical surface into space curves. A shape space of such artangements is constructed equipped with curve registration techniques to quantify shape resemblance and dissimilarity. Although the metric measures global shape differences, deformation energies allow us to identify the regions where anatomical differences and similarities are most pronounced. The metric is applied to the analysis of configurations of sulcal curves associated with the left and right decompositions into space curves of surfaces representing the entire left hemisphere.

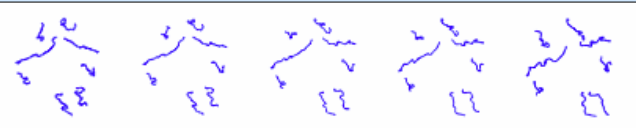

Figure 1. A geodesic between configurations of sulcal lines.

\section{Representation and Metric}

A configuration of $n$ parametric curves $\alpha_{j}:[0,1] \rightarrow R^{3}, 1 \leq j \leq n$, is represented by a 3xn matrix A whose entries are the components $\alpha_{i j}:[0,1] \rightarrow \mathrm{R}(\mathrm{i}=1,2,3)$ of the curves. The Sobolev inner product $\left.<\mathrm{f}, \mathrm{g}\rangle_{1}=\mathrm{a}\right] \mathrm{f}(\mathrm{s}) \mathrm{g}(\mathrm{s}) \mathrm{ds}+\mathrm{b} \int \mathrm{f}^{\prime}(\mathrm{s}) \mathrm{g}$ '(s)ds

(1)

$$
\langle A, B\rangle=\sum\left\langle\alpha_{i j}, \beta_{i j}\right\rangle_{1} .
$$

This metric accounts for shape geometry to first order, but higher order metrics can be defined similarly. As in classical Procrustes alignment, we first place the centroid of the configuration at the origin and scale the configuration so that $\|\mathrm{A}\|=1$. This makes the representation insensitive to translations and scale and restricts A to the infinite-dimensional unit sphere (about zero) in the subspace of centered matrices. To calculate the optimal orthogonal alignment between normalized matrices $\mathrm{A}$ and $\mathrm{B}$, let $\mathrm{M}$ be the $3 \times 3$ matrix whose $(\mathrm{i}, \mathrm{j})$ entry is the scalar $\sum_{1}<\alpha_{\mathrm{il}}, \beta_{\mathrm{jil}}>$. If $\mathrm{M}=\mathrm{USV}^{\mathrm{T}}$ is a singular value decomposition of $\mathrm{M}$, then the optimal orthogonal alignmen of $\mathrm{B}$ relative to $\mathrm{A}$ is given by $\mathrm{B}=\mathrm{M}-\mathrm{B}$. Moreover, the geodesic shape distance is given by $\mathrm{d}(\mathrm{A}, \mathrm{B})=\arccos \omega$, where $\omega=$ trace( $\mathrm{S})$, and the geodesic deformation is realized by the path

(2) $\quad \Lambda(t)=\cos (\omega t) A+\sin (\omega t) v(A, B)$,

with $v(A, B)=\left(B^{\prime}-\omega \mathrm{A}\right) /\left\|B^{*}-\omega \mathrm{A}\right\|$.

The geodesic deformation energy is given by $\mathrm{E}=\int\left\|\Lambda^{\prime}(\mathrm{t})\right\|^{2} \mathrm{dt}=\mathrm{d}^{2}(\mathrm{~A}, \mathrm{~B})$.

\section{Decomposition of Surfaces}

To represent a spherical surface with a collection of space curves, we first construct a minimal distortion parametrization $\alpha: \mathrm{S}^{2} \rightarrow \mathrm{R}^{3}$ with the techniques of [1,2], and then decompose the surface using a collection of parallels

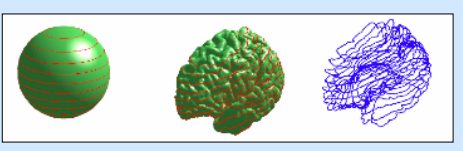

Figure 2. Decomposition of a surface into space curves

\section{Shape Alignment}

In the case of sulcal lines, the correspondence between curves in different configurations is established using be optimal elastic

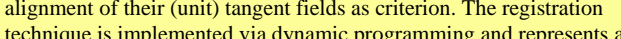
variant of methods previously used for single curves [2].

Parametric spherical surfaces were aligned with the techniques of $[1,3]$ and a common set of parallels of $\mathrm{S}^{2}$ was used to decompose them in a compatible manner, as indicated in Figure 2.

\section{Examples of Geodesics}

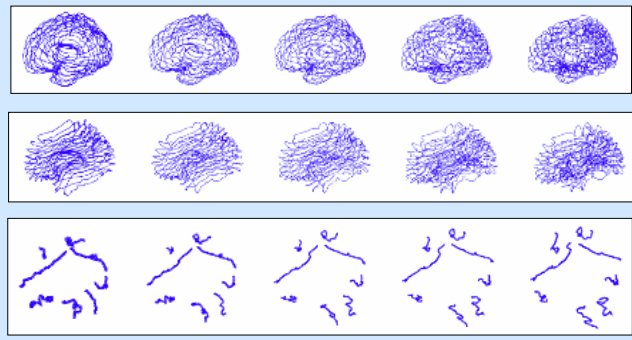

Fig. 2. Geodesic interpolations between aligned arrangements of curves.

Acknowledgements Acknowledgements
This work was supported in part by the National Science Foundation (NSF)
grant CCF-0514743.

\section{Local Shape Differences}

The energy of the geodesic path (2) is given by $E(\Lambda)=\int_{0}^{1}\left\|\Lambda^{\prime}(t)\right\|^{2} d t=d^{2}(A, B)$ Thus, the function

$$
\rho_{j}(s)=\frac{1}{d^{2}(A, B)} \sum_{i} \int_{0}^{1}\left(a\left|\Lambda_{i j}^{\prime}(t)\right|^{2}+b\left|\partial_{s} \Lambda_{i j}^{\prime}(t)\right|^{2}\right) d t
$$

can be interpreted as the energy density along the jth curve of the arrangement, which gives a measurement of the local contributions to the total shape distance.

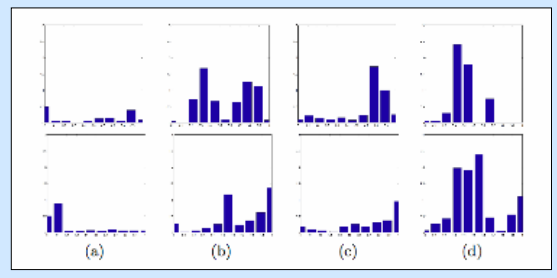

Fig. 3. Geodesic deformations between the left and right hemispheres of 2 subjects: (a) calcarine; (b) central; (c) superior frontal; (d) superior temporal.

\section{Left vs. Right Hemispheres}

\begin{tabular}{|c|c|c|c|}
\hline $\mathbf{a}$ & $\mathbf{b}$ & $\mathbf{k}$ & Correct Classification \\
\hline 1 & 0 & 1 & $100 \%$ \\
\hline 1 & 0 & 3 & $100 \%$ \\
\hline 1 & 0 & 5 & $100 \%$ \\
\hline 1 & 1 & 1 & $79 \%$ \\
\hline 1 & 1 & 3 & $75 \%$ \\
\hline 1 & 1 & 5 & $92 \%$ \\
\hline
\end{tabular}

Experiments with 24 configurations of 4 sulcal curves representing the left and right hemispheres of 12 subjects, with weights a and $b$ for the Sobolev metric. Decisions were based on the k-nearest neighbor classifier and a leave-one-out approach was used.

\section{References}

[1] X. Liu, J.C. Bowers, and W. Mio, Parametrization, Alignment and Shape of [2] W. Mio, J.C. Bowers, and X. Liu, Shape of Elastic Strings in Euclidean Space: An Infinite-Dimensional Familhy of Metrics, FSU Technical Report, 2007. [3] E. Praun and H. Hoppe, Spherical Parametrization and Remeshing, AMC 